\title{
Reprodução da ave piru-piru (Haematopus palliatus, Temminck 1820, Haematopodidae) no litoral sul do Estado de São Paulo, Brasil
}

\author{
Edison Barbieri ${ }^{1,2}$ \& Roberta Tonolli Chiavone Delchiaro ${ }^{I}$ \\ ${ }^{1}$ Instituto de Pesca - APTA - SAA/SP. \\ Av. Prof. Besnard s/n, Morro do São João, CEP 11990-000, Cananéia, SP, Brasil \\ ${ }^{2}$ Autor para correspondência: Edison Barbieri,e-mail: edisonbarbieri@yahoo.com.br
}

BARBIERI, E. \& DELCHIARO, R.T.C. Nesting of the American Oystercatcher Haematopus palliatus Temminck 1820, Haematopodidae in the southern coast of São Paulo State, Brazil. Biota Neotrop. 9(4): http:// www.biotaneotropica.org.br/v9n4/en/abstract?short-communication+bn02609042009.

\begin{abstract}
This note reports the occurrence and nesting of Haematopus palliatus in southern coast of São Paulo State. Data were obtained from direct observations of nests between August/2005 and December/2008. A total of 22 nests, every one with two eggs, were monitored, including 20 at Ilha Comprida and two at Ilha do Cardoso. Of these, 13 produced at least one chick. These are the first data about breeding of Haematopus palliatus in southern São Paulo State.

Keywords: American Oystercatcher, Brazil, Ilha Comprida, Ilha do Cardoso, Haematopus palliatus, Nesting, São Paulo.
\end{abstract}

BARBIERI, E. \& DELCHIARO, R.T.C. Reprodução da ave piru-piru (Haematopus palliatus, Temminck 1820, Haematopodidae) no litoral sul do estado de São Paulo, Brasil. Biota Neotrop. 9(4): http://www. biotaneotropica.org.br/v9n4/en/abstract?short-communication+bn02609042009.

Resumo: Esta nota registra a ocorrência e nidificação do Haematopus palliatus na costa sul do Estado de São Paulo. Os dados foram obtidos de observações diretas dos ninhos no período de agosto de 2005 a dezembro de 2008. Um total de 22 ninhos, cada um com dois ovos, foram monitorados, incluindo 20 na Ilha Comprida e dois na Ilha do Cardoso. Destes, 13 chegaram a produzir filhotes. Este é um registro inédito sobre a reprodução de Haematopus palliatus na costa sul de São Paulo.

Palavras-chave: Brasil, Haematopus palliatus, Ilha Comprida, Ilha do Cardoso, Piru-piru, reprodução, São Paulo. 


\section{Introdução}

Haematopus palliatus é uma ave de praia registrada ao sul da América do Norte, na costa Pacifica e Atlântica, e em toda a costa da América do Sul (Hayman et al.1986); incluindo toda a costa brasileira, onde nidifica (Sick 1997). Esta espécie é restrita à beira mar, encontrada em costões rochosos expostos à arrebentação e praias arenosas (Hayman et al. 1986). No Brasil é uma ave observada em todos os estados, com maior abundância no Rio Grande do Sul (Sick 1997). Alimenta-se principalmente de gastrópodes, ostras, cracas e lamelibrâmquios, caranguejos e outros invertebrados (Nol et al. 1984, Hayman et al. 1986, Sick 1997), forrageando principalmente em praias arenosas, costões rochosos e planícies intermareais (Nol 1989). São encontrados geralmente forrageando em pares isolados (Figura 1), mostrando forte territorialidade durante o período reprodutivo (Cadman 1979).

A postura é, em geral, de dois ovos, ocasionalmente chegando a quatro (Lauro \& Burger 1989). Estes são marrom claro com pontos escuros e outras manchas, medindo aproximadamente $5,0 \mathrm{~mm}$ por $38 \mathrm{~mm}$ (Nol et al. 1984). Para disfarçar os ovos, dependendo do local, os adultos adicionam seixos quebrados aos ninhos (Nol 1989). Ambos os membros do par reprodutor incubam os ovos e cuidam dos filhotes (Sick 1997).

Os ninhegos podem estar ativos dentro de 24 horas após nascerem, mas levam até 60 dias para que seus bicos tornem-se fortes o bastante para abrir suas próprias presas (Nol et al. 1984, Lauro \& Burger 1989). Os filhotes podem permanecer por até seis meses com os respectivos pares reprodutores, os quais os protegem vigorosamente (Lauro \& Burger 1989). Para confundir os predadores, os adultos se fingem de feridos para atrair a atenção para longe do ninho ou, ainda, de estarem chocando em outro local onde não há nenhum ninho (Sick 1997). Estas aves alcançam a maturidade sexual após um ano (Hayman et al. 1986) e os adultos podem viver dez anos ou mais (Nol 1984).

Não há trabalhos realizados sobre a reprodução e nem estimativa da população de $H$. palliatus no Brasil. Em São Paulo, no sudeste brasileiro, também não existem trabalhos que estimem o tamanho populacional de $H$. palliatus, embora a espécie esteja listada como "Vulnerável" para este Estado (Decreto N ${ }^{\circ} 53.494$, de 2 de outubro de 2008), ou ainda, que indiquem áreas de nidificação. No presente trabalho apresento as primeiras informações sobre a nidificação de H. palliatus para o litoral paulista.

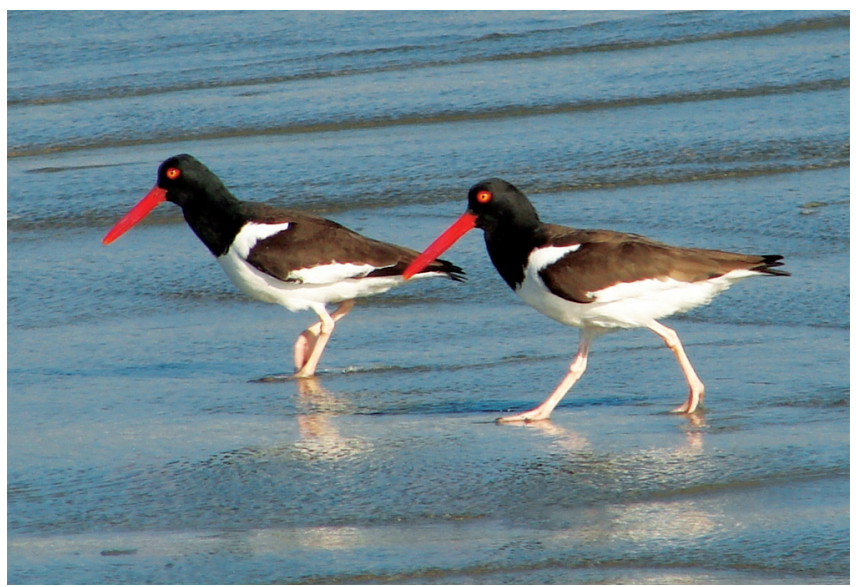

Figura 1. Par reprodutor de Haematopus palliatus, forrageando na praia da Ilha Comprida, no litoral sul do estado de São Paulo.

Figure 1. Breeding pair of Haematopus palliatus, feeding at Ilha Comprida beach, southern coast of São Paulo State.

\section{Material e Métodos}

As observações foram realizadas semanalmente nos sítios de nidificação de H. palliatus nas Ilhas Comprida e do Cardoso, no litoral extremo sul do Estado de São Paulo. Na Ilha Comprida foram percorridas as praias e áreas partindo da parte sul em direção ao norte, em uma extensão de $70 \mathrm{~km}$ (Figura 2). Na Ilha do Cardoso caminhou-se da praia do Pereirinha até a de Itacuruça, uma faixa de $8 \mathrm{~km}$.

Na Ilha Comprida, as observações e busca dos ninhos estenderamse de agosto de 2005 a dezembro de 2008, totalizando 80 visitas semanais. A Ilha do Cardoso foi visitada somente nos anos de $2007 \mathrm{e}$ 2008, durante os mesmos meses amostrados para a Ilha Comprida.

Em cada sítio reprodutivo localizado foi determinado semanalmente a quantidade de ovos por ninho, o comprimento e a largura (em milímetros, paquímetro com precisão $0,1 \mathrm{~mm}$ ) e a massa (em gramas, balança tipo dinamômetro com precisão $0,1 \mathrm{~g}$ ) dos ovos. As medidas dos ovos - comprimento total $(\mathrm{cm})$, largura $(\mathrm{cm})$ e massa (g) - foram tomadas usando-se paquímetro (Mitutoyo de $150 \mathrm{~mm}$, precisão $0,1 \mathrm{~mm}$ ) e balança tipo dinamômetro (Pesola de $100 \mathrm{~g}$, precisão $0,1 \mathrm{~g}$ ). Filhotes com 15 dias de vida tiveram sua massa corpórea aferida (g) e tomadas medidas do comprimento do cúlmen exposto (cm) conforme Sick (1997).

Dois ninhos na Ilha Comprida foram monitorados diariamente entre 15 de agosto até 15 de dezembro de 2008 para avaliar o tempo de incubação e de sobrevivência.

\section{Resultados}

Entre agosto de 2005 e dezembro de 2008 foram registrados um total de 22 ninhos, incluindo 20 na Ilha Comprida e dois na Ilha do Cardoso. Os ninhos consistiram em depressões rasas escavadas em partes mais elevadas de praias arenosas acima da linha da maré de sizígia (Figura 3). Em 2005, na Praia da Ilha Comprida e na Ilha do Cardoso, foram registrados dos quatro pares reprodutores

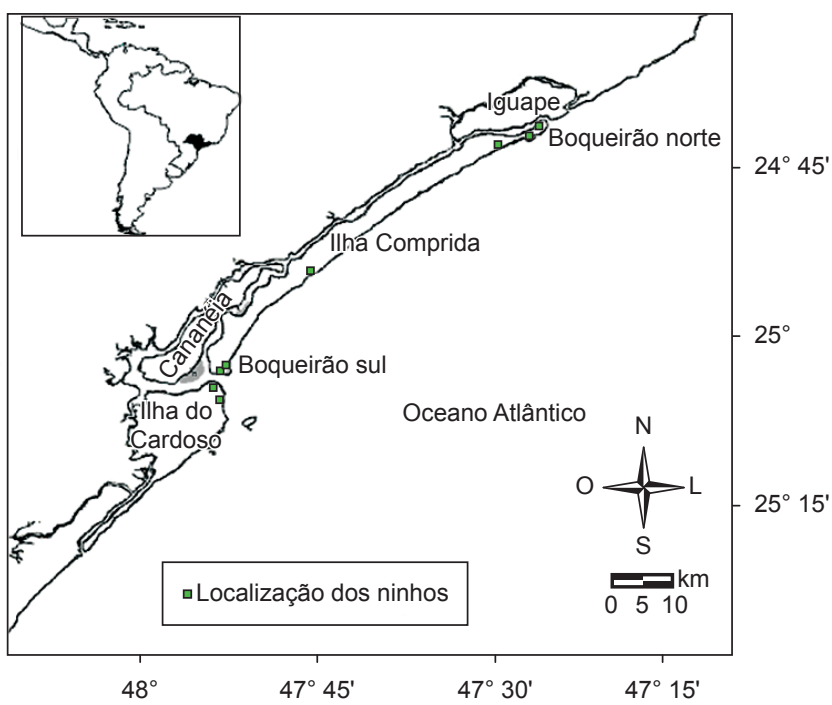

Figura 2. Localização dos ninhos de Haematopus palliatus, envolto pela planta de praia (Blutaparon portulacoides) na Ilha Comprida e Ilha do Cardoso, áreas amostradas no litoral sul do estado de São Paulo.

Figure 2. Locality of Haematopus palliatus nests, involved for the beach plant (Blutaparon portulacoides), at Ilha Comprida and Ilha do Cardoso, sampled areas in the south coast of São Paulo State. 
de H. palliatus nidificando nas dunas, seis em 2006, oito em 2007 e quatro em 2008. Também foram encontrados dois ninhos de H. palliatus, na praia do Pereirinha na Ilha do Cardoso, com dois ovos cada.

Nas Ilhas Comprida e do Cardoso a época de reprodução foi de outubro a janeiro, durante os quatro anos estudados. A postura ocorreu em meados de outubro e os primeiros filhotes foram observados em novembro. O tempo de incubação variou de 25 a 30 dias para os dois ninhos da Ilha Comprida monitorados diariamente.

Os ninhos eram cavidades rasas escavadas na areia, onde eram depositados dois ovos de coloração marrom-clara com pequenas manchas pretas distribuídas irregularmente, bem camuflados (Figura 3).

O comprimento dos ovos variou entre 50,9 a $51,9 \mathrm{~mm}$, com largura entre 3,40 a $3,68 \mathrm{~cm}$ e peso entre 9,2 a $10 \mathrm{~g}(\mathrm{n}=22)$ (Tabela 1$)$.

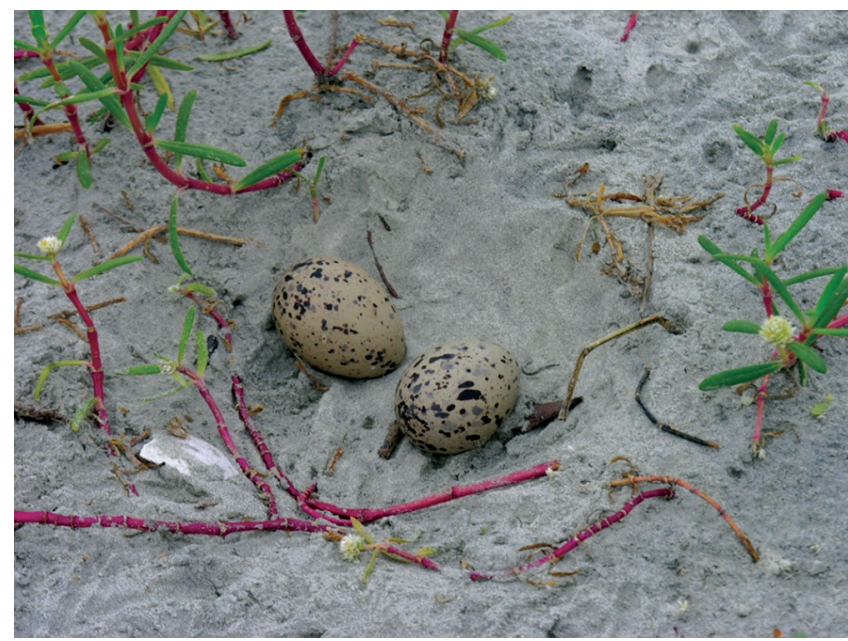

Figura 3. Ovos de Haematopus palliatus na Ilha Comprida, litoral sul do estado de São Paulo.

Figure 3. Eggs of Haematopus palliatus at Ilha Comprida, southern coast of São Paulo State.
O comprimento do cúlmen exposto de 13 filhotes de 15 dias oscilou entre 18,3 a 21,2 mm e o peso entre 50 a $53 \mathrm{~g}$ (Tabela 2). Os ovos dos ninhos da Ilha do Cardoso mediram entre 51,2 e 50,8 mm, com largura de 33,6 e 35,4 mm e peso de 9,80 e 10 gramas, respectivamente. Estes ninhos não obtiveram sucesso na eclosão dos ovos.

Em apenas 13 ninhos houve a eclosão dos ovos com desenvolvimento de um único filhote por ninho (Figura 4). Em um ninho na Ilha Comprida observou-se Caracara plancus predando os ovos do H. palliatus. Possivelmente cães e gatos domésticos, comuns nas comunidades caiçaras de ambas as ilhas, e associados a casas de veraneio, também possam predar os ovos e filhotes.

\section{Discussão}

Embora não se tenha registro de estudos populacionais de H. palliatus para o estado de São Paulo, esta espécie é listada como

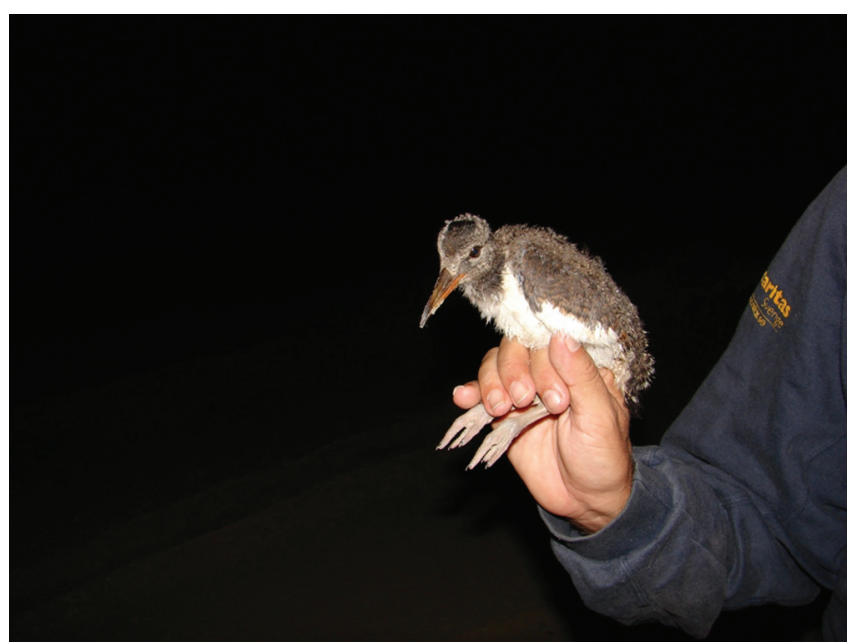

Figura 4. Filhote de Haemantopus palliatus com 15 dias na Ilha Comprida, litoral sul do estado de São Paulo.

Figure 4. Chick of Haemantopus palliatus 15 days old at Ilha Comprida, southern coast of São Paulo State.

Tabela 1. Médias das medidas dos ovos (em mm) de Haemantopus palliatus (Temminck 1820, Haematopodidae) registrados entre 2005 e 2008 na Ilha Comprida e Ilha do Cardoso, litoral sul do estado de São Paulo. Os números entre parêntesis, à direita das médias apresentadas, representam os respectivos desvios-padrão.

Table 1. Averages of the measures of eggs (in mm) of Haemantopus palliatus (Temminck 1820, Haematopodidae) recorded from 2005 to 2008 at Ilha Comprida and Ilha do Cardoso, southern coast of São Paulo State. The numbers between parentheses, to the right of the presented averages, represent respective standard deviation.

\begin{tabular}{lcccc}
\hline \multicolumn{1}{c}{ Ano } & $\mathbf{2 0 0 5}(\mathbf{n}=\mathbf{4})$ & $\mathbf{2 0 0 6}(\mathbf{n}=\mathbf{6})$ & $\mathbf{2 0 0 7}(\mathbf{n}=\mathbf{8})$ & $\mathbf{2 0 0 8}(\mathbf{n}=\mathbf{4})$ \\
\hline Comprimento & $52,8(0,18)$ & $51,3(0,16)$ & $52,1(0,12)$ & $52,2(0,10)$ \\
Largura & $41,1(0,14)$ & $40,5(0,13)$ & $41,7(0,14)$ & $41,4(0,05)$ \\
Peso & $12,4(0,40)$ & $12,12(0,15)$ & $12,20(0,17)$ & $12,13(0,03)$ \\
\hline
\end{tabular}

Tabela 2. Médias das medidas dos ninhegos de 15 dias de Haemantopus palliatus (Temminck 1820, Haematopodidae) registrados no litoral sul do estado de São Paulo. Os números entre parêntesis, à direita das médias apresentadas, indicam os desvios-padrão.

Table 2. Averages of the measures of chicks (15 days old) of Haemantopus palliatus (Temminck 1820, Haematopodidae) recorded in the south coast of São Paulo State. The numbers in parentheses, to the right of the presented averages, mean the standard deviation.

\begin{tabular}{ccrrr}
\hline Ano & $\mathbf{2 0 0 5}(\mathbf{n}=\mathbf{2})$ & $\mathbf{2 0 0 6}(\mathbf{n}=\mathbf{4})$ & $\mathbf{2 0 0 7}(\mathbf{n}=\mathbf{4})$ & $\mathbf{2 0 0 8}(\mathbf{n}=\mathbf{3})$ \\
\hline Comprimento $(\mathrm{cm})$ & $1,97(0,20)$ & $1,94(0,12)$ & $2,05(0,06)$ & $2,11(0,09)$ \\
Peso (g.) & $51,5(2,12)$ & $50,55(0,77)$ & $51(0,90)$ & $52,63(1,20)$ \\
\hline
\end{tabular}


"Vulnerável" (Decreto No 53.494, de 2 de outubro de 2008). Esta categoria refere-se às espécies com alto risco de extinção em médio prazo, ameaçadas por alterações ambientais significativas, diminuição da área de distribuição ou declínio populacional ou ainda da diminuição da sua área de distribuição. A baixa produtividade da espécie e sua pequena população no litoral sul de São Paulo (4-8 pares reprodutores em $72 \mathrm{~km}$ de praia na Ilha Comprida) confirmam sua categorização como ameaçada.

Ao longo das quatro temporadas reprodutivas monitoradas, três sítios foram re-utilizados por $H$. palliatus em estações sucessivas. Apesar da ausência de marcações individuais nos adultos, a ocupação dos mesmos territórios em anos consecutivos, somada ao fato da espécie ser territorial, principalmente durante o período reprodutivo, sugere a fidelidade dos pares reprodutores aos sítios de nidificação ao longo dos anos, o que necessita ser confirmado

A especulação imobiliária ao longo do litoral paulista para a construção de residências e outros empreendimentos relacionados com veraneio (turismo), e o grande número de pessoas e veículos utilizando as praias, são a principal ameaça às dunas e praias na Ilha Comprida (Barbieri \& Tavares 2008). Haematopus palliatus dependem dos ambientes costeiros para reprodução, entre os quais dunas vegetadas (Shields \& Parnel, 1990), o que foi observado também para Charadrius collaris em São Paulo (Barbieri \& Mendoça, 2005, Barbieri \& Pinna, 2005).

Em um estado onde a faixa costeira foi intensamente ocupada e com poucas praias que combinam baixa perturbação humana e de animais domésticos com a presença de vegetação de praia (Blutaporon portulacoides, Evolvurus pusillus, Ipomea littoralis, Ipomea pés-caprae) e de dunas (Cladium mariscus, Eleocharis capita, Spartina ciliata, Sternotaphrum secundatum), mesmo em unidades de conservação, é óbvia a necessidade de preservar este habitat onde ele ainda ocorre para minimizar as ameaças às espécies que dele dependem, especialmente aves costeiras que nele nidificam.

\section{Agradecimentos}

Agradecemos aos funcionários do Núcleo de Desenvolvimento do Litoral do Instituto de Pesca: Onésio Veríssimo, Antônio Pires e Gilson Calasans, pelo apoio durante várias etapas desse trabalho.

\section{Referências}

BARBIERI, E. \& MENDONÇA, J.T. 2005. Distribuition and abundance of Charadridae at Ilha Comprida, São Paulo State, Brazil. J. Coastal Res. 21(2):e1-e10.

BARBIERI, E. \& PINNA, F.V. 2005. Distribuição da Batuíra-de-coleira (Charadrius collaris) durante o período de 1999 a 2001 na praia da Ilha Comprida. Rev. Bras. Ornitol. 13(2):25-31.

BARBIERI, E. \& TAVARES, E.T. 2008. The birds at Ilha Comprida beach (São Paulo State, Brazil): a multivariate approach. Biota Neotrop. 8(3): http:// www.biotaneotropica.org.br/v8n3/en/abstract?article+bn00408032008 (ultimo acesso em 19/09/2009).

CADMAN, M. 1979. Territorial behaviour in American Oystercatches Haematopus palliatus. Wader. Study Group Bull. 27(1):40-41.

HAYMAN, P., MARCHANT, J. \& PRATER, T. 1986. Shorebirds an identification guide. Houghtton Mifflin Company, Boston, 412 p.

LAURO, B. \& BURGER, J. 1989. Nest-site selection of American Oystercatchers (Haematopus palliatus) in salt marshes. Auk 106(2):185-192.

NOL, E. 1984. Reproductives strategies on the Oystercatcher (Aves: Haematopodidae). PhD. thesis, University of Toronto, Toronto.

NOL, E., BAKER, A.J. \& CADMAN, M.D. 1984. Clutch initiation dates, clutch size, and egg size of the American Oystercatcher in Virginia. Auk. 101(4):855-867.

NOL, E. 1989. Food Supply and Reproductive Performance of the American Oystercatcher in Virginia. Condor. 91(2):429-435.

SICK, H. 1997. Ornitologia Brasileira. Ed. Nova Fronteira, Rio de Janeiro.

SHIELDS, M.A. \& PARNELL, J.F. 1990. Marsh nesting by American Oystercatchers in north Carolina. J. Field Ornithol. 61(4):431-433

Recebido em 04/08/09

Versão reformulada recebida em 28/10/09

Publicado em 18/11/09 